REVIEW

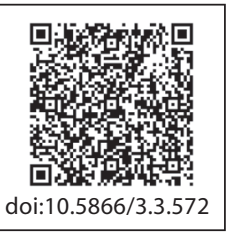

\title{
Recent advances of Pendulum Appliance for Effective Molar Distalization
}

\author{
Lalitha $\mathrm{Ch}^{1}$, Vasumurthy $\mathrm{S}^{2}$, Vikasini $\mathrm{K}^{3}$
}

Department of Orthodontics

Kamineni Institute of Dental Sciences

Narketpally, Nalgonda Dist. A.P.

\section{Sr. Lecturer ${ }^{1}$}

Professor \& HOD ${ }^{2}$

Post graduate student ${ }^{3}$

\section{Article Info}

Received: April 12, 2011

Review Completed: May, 15, 2011

Accepted: June, 17, 2011

Available Online: October, 2011

(c) NAD, 2011 - All rights reserved

\begin{abstract}
:
Molar distalization is the process of moving the terminal molars distally. It is one of the non extraction treatment modalities which is frequently followed in the regular clinical practice. To achieve a compliance free molar distalization, Hilger's Pendulum appliance is the best choice. It has undergone many modifications since its introduction to best suit a clinical situation. This article reviews the Pendulum appliance and its various modifications in brief.
\end{abstract}

\section{Introduction:}

Class II malocclusions can be corrected by combinations of restriction or redirection of the maxillary growth, distal movement of the maxillary dentition, mesial movement of the mandibular dentition, and enhancement or redirection of the mandibular growth. To establish a class I molar relationship and create space in the lateral segments for canines or premolars, in non extraction treatment modalities, distalization of maxillary first molars is the choice. $^{1}$

Many distalizing mechanisms were used since then such as the Wilson bimetric distalizing arch (Rocky Mountain Orthodontics, Denver, CO), the Jones jig, the acrylic cervical occipital appliance (ACCO), and Class II elastics. A significant problem with these approaches is that they require patient cooperation. ${ }^{2}$

To diminish cooperation, various distalizing systems have been developed that require less

Email for correspondence:

drplalithasunil@gmail.com
Key words: Molar distalization, Pendulum appliance, Pendex

patient cooperation. Most of these appliances have used fixed Nance buttons with either open coil springs or repelling magnets. One such appliance is the Pendulum. ${ }^{2}$

An ideal intraoral molar-distalization appliance should meet the following criteria: ${ }^{3}$

- Minimal need for patient compliance

- Acceptable esthetics and comfort

- Minimal loss of anterior anchorage (as evidenced by axial proclination of the incisors)

- Bodily movement of molars to avoid undesirable side effects, lengthening of treatment, and unstable results

- Minimal chairtime for placement and reactivations

Among the distalizing methods introduced, the Hilgers Pendulum Appliance seems to satisfy these requirements. Even this device, however, can produce unwanted tipping of the maxillary molars during distalization. ${ }^{3}$ 


\section{PENDULUM APPLIANCE:}

The Pendulum, was introduced by Hilgers ${ }^{4}$ in 1992. The Pendulum appliance consists of a palatal Nance component with rests that are bonded to the occlusal surface of the first and/or second premolar teeth. The distalizing mechanism consists of bilateral helical springs composed of titanium molybdenum alloy. The Pendulum appliance is unique in that it does not rely on coil springs for its action. Instead, 0.032 "TMA springs deliver a continuous force against the maxillary first molars.

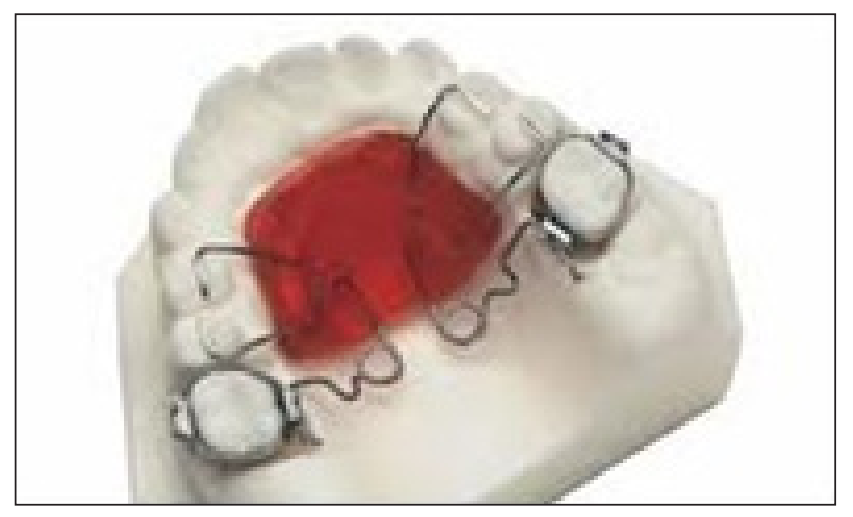

According to Hilgers, "the appliance produces a broad, swinging arc-or pendulum-of force from the midline of the palate to the upper molars. The springs of the appliance produce 200 to $250 \mathrm{~g}$ of force in a swinging arc movement, hence the name Pendulum.

Hilgers calls for preactivation of the appliance by bending the springs to a $90^{\circ}$ angle. About a third of the bend is lost in the insertion, resulting in a $60^{\circ}$ activation, or $250 \mathrm{~g}$ of distalizing force. Although the spring pressure must be monitored constantly, further activations are not usually required. The springs can produce about $5 \mathrm{~mm}$ of distal movement in three to four months. Loss of anchorage is minimal: $1.5 \mathrm{~mm}$ in the premolar area and about $1-2^{\circ}$ of proclination of the maxillary incisors

\section{Clinical indications of Maxillary molar Distalization:}

Molar distalization in the maxillary arch is an important part of the therapeutical armaments in everyday orthodontic practice.
Clinical indications can be classified as:

a) Skeletal problems

-maxillary protrusion

-maxillary protrusion associated with mandibular retrusion

b) Dentoalveolar problems

-mesial position of the upper dental arch

-tooth size /arch length discrepancy at the upper arch

c) Dental problems

-mesial positioning of the maxillary first molars (due to caries, early resorption, or severe infraocclusion of second deciduous molars)

Pendulum appliance is contraindicated in patients with:

- Skeletal or dental open bites,

- High mandibular plane angles,

- Excessive lower facial height, or

- Proclined maxillary incisors.

Advantages of Pendulum appliance over other distalizing appliances:

1. The need for minimal patient cooperation is one of the most desirable qualities of the Pendulum appliance when compared with other appliances such as the headgear, the ACCO, and the Wilson distalizing arch.

2. Only one activation period is mostly necessary to achieve results. Most distalizing appliances, including headgears, the ACCO, and Wilson mechanics, require some sort of reactivation or titration of forces to effect a change.

3. Pendulum does not require intermaxillary elastics for anchorage support, as required in the Wilson distalizing system. So loss of anchorage in the mandibular arch is not a concern.

\section{Side effects :}

Distal molar tipping and anchorage loss at the incisors.

Ghosh and Nanda ${ }^{5}$ evaluated 41 patients treated with pendulum appliance and found $57 \%$ of the average class II correction was molar distalization and $43 \%$ maxillary first premolar and anterior anchorage 
loss. The patients in this sample also demonstrated a $2.8 \mathrm{~mm}$ average increase in lower facial height.

Joseph and Butchart ${ }^{2}$ noted an average of $5.1 \mathrm{~mm}$ of molar distalization, $3.7 \mathrm{~mm}$ anterior movement of the maxillary incisors, an average distal tipping of maxillary first molar of $15.7^{\circ}$ and an average flaring of the maxillary incisor of $4.9^{\circ}$ in their study.

The results of Bussick and McNamara ${ }^{6}$ study suggests that the pendulum appliance is effective in moving maxillary molars posteriorly during orthodontic treatment. For maximum maxillary first molar distalization with minimal increase in lower facial height, this appliance is used most effectively in patients with deciduous maxillary second molars for anchorage and unerupted permanent maxillary second molars.

\section{Modifications of Pendulum appliance: PENDEX appliance ${ }^{4}$ :}

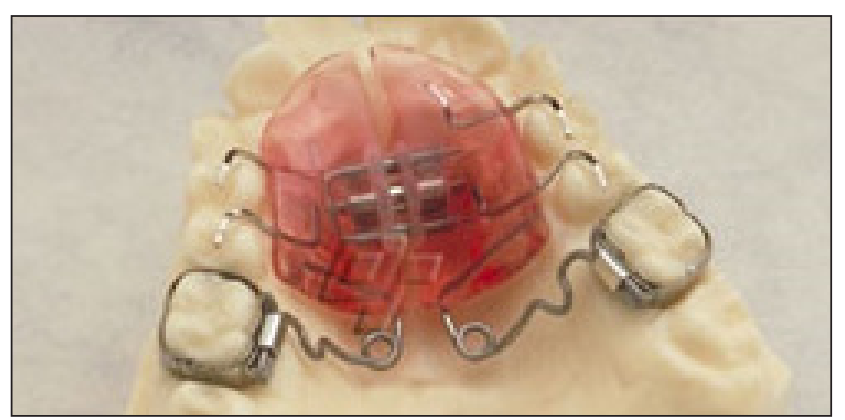

Pendex appliance was given by Hilgers. The design of the Pendex appliance is essentially the same as the Pendulum, except for the addition of a palatal expansion screw in the midline. It is used in instances where there is tendency towards transverse maxillary constriction as in patients with class II malocclusion.

\section{T-REX appliance:}

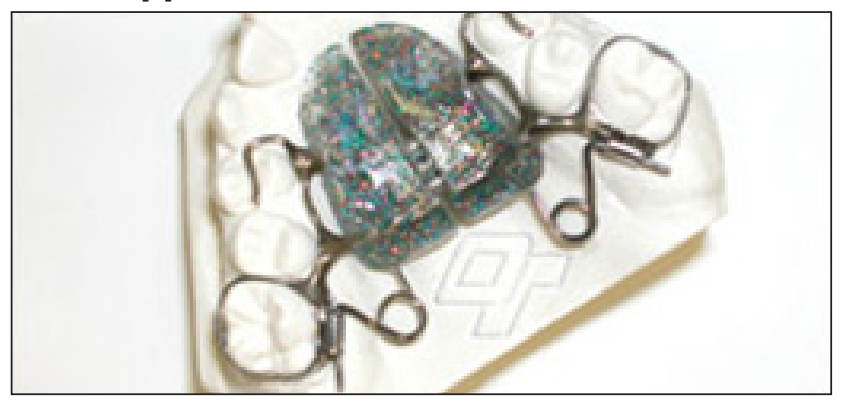

This design features two wires that extend from the palatal acrylic and are soldered to the lingual aspect of the maxillary first molars. These wires provide additional stability to the appliance during the expansion phase; they are severed or removed when the molar distalization phase is initiated.

\section{Hilgers Phd appliance:}

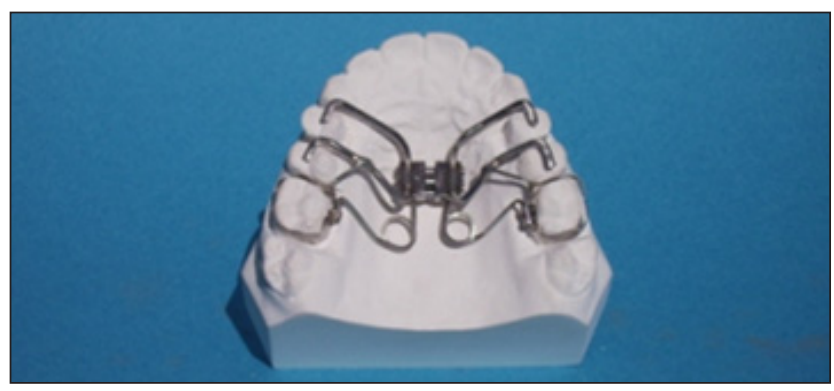

Another appliance in the PENDULUM family is the Phd appliance. This version features an all-metal design with no acrylic plate. The primary advantage being comfort and improved hygiene for the patient. Anchorage consists of banded first premolars with stabilizing wires from the premolars to the molar bands. This appliance allows for phasing the treatment if desired, with palatal expansion first, followed by sectioning of the holding wires and distalizing of the molars. The pendulum springs in the Phd appliance insert into sheaths welded or soldered to the palatal side of the expansion screw housing. This configuration allows the clinician to remove the springs, either before palatal expansion is started or for adjustments/reactivation of the springs during the molar distalizing phase.

\section{Pendulum appliance with maxillary molar root uprighting bends ${ }^{7}$ :}

The Pendulum appliance was modified by incorporating an uprighting bend into the distalizing spring during the second phase of treatment to avoid excessive distal tipping of the maxillary molars which was observed when the conventional pendulum appliance was used. The Pendulum appliance with uprighting bends led to reduced molar tipping without significantly changing the effects of the Pendulum appliance, with the exceptions of $0.62 \mathrm{~mm}$ more anchorage loss of the maxillary incisor edge and increased treatment time. 


\section{The Modified Pendulum Appliance/M-Pendulum ${ }^{3}$ :}

The horizontal Pendulum omega loop is inverted, which will allow bodily movement of both the roots and crowns of the maxillary molars. Once distal molar movement has occurred, the loop can be activated simply by opening it. The activation produces buccal and/or distal uprighting of the molar roots and thus a true bodily movement rather than a simple tipping or rotation.

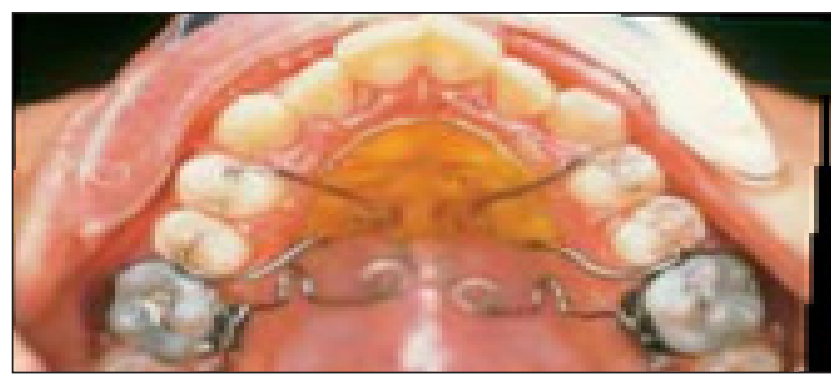

The Modified Pendulum Appliance with Removable Arms ${ }^{8}$ :

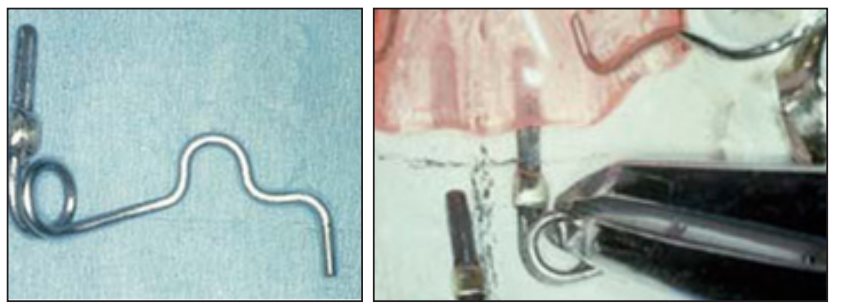

The active arms of the Pendulum appliance are inserted into the acrylic sheaths of the Nance button. These removable arms can be reactivated during treatment without debonding and rebonding the occlusal rests of the Nance button. Distal molar movement can then be more precisely controlled than by opening the horizontal loops in the mouth. The conventional Pendulum or M-Pendulum produces about $5 \mathrm{~mm}$ of distalization in three to four months. With the removable arms, distal movement can be continued at a rate of about $1.5 \mathrm{~mm}$ per month for as long as necessary.

\section{Franzulum Appliance?:}

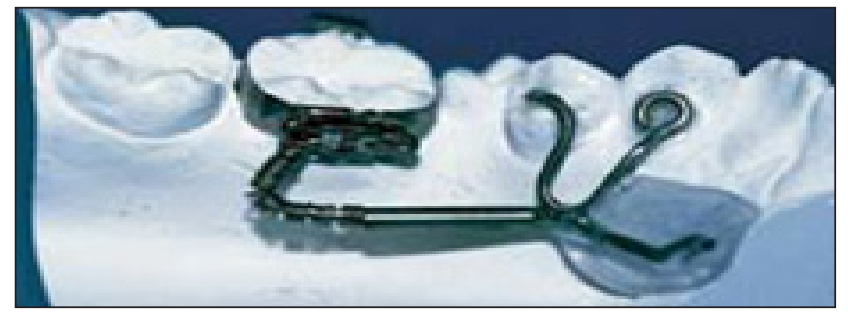

It is the modification of the pendulum appliance to distalize the mandibular molars. It consists of an anterior anchorage unit of an acrylic button, positioned lingually and inferiorly to the mandibular anterior teeth, and extending from the mandibular left canine to the mandibular right canine. Rests on the canines and first premolars are made from .032" stainless steel wire. Tubes between the second premolars and first molars receive the active components. The posterior distalizing unit uses nickel titanium coil springs, about $18 \mathrm{~mm}$ in length, which apply an initial force of 100-120g per side. A J-shaped wire passing through each coil is inserted into the corresponding tube of the anchorage unit; the recurved posterior portion of the wire is engaged in the lingual sheath of the mandibular first molar band.

\section{The Mini-Distalizing Appliance (MDA) ${ }^{10}$}

It is another modified version in this family of molar distalizers. A hybrid appliance that incorporates the best features of both the Pendulum and the Compact RPE (rapid palatal expander), it is an excellent choice to expand the maxilla, distalize upper molars, create room for erupting cuspids, and unlock the anterior occlusion. It is truly a threedimensional appliance.

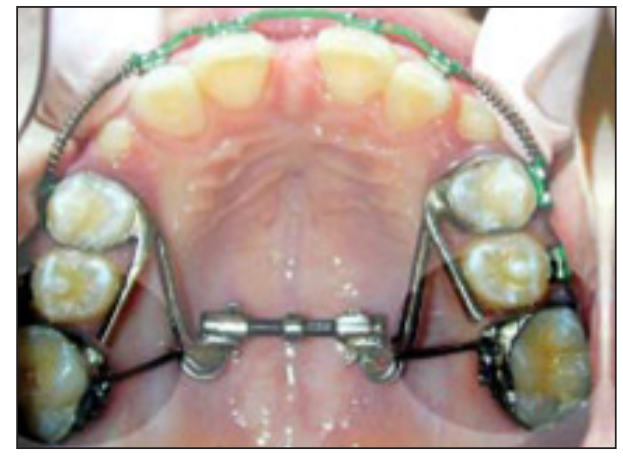

The MDA's small, rigid design affords exceptional patient comfort without compromising effectiveness. Because it is a toothborne appliance with no palatal coverage, the problems of tissue impingement and access for hygiene are greatly reduced or eliminated.

\section{Pendulum $\mathbf{K}^{11}$ :}

Incorporates a distal screw into the Nance button and the initial application of an uprighting and a toein bend in the region of the pendulum springs and thus preventing the potential side effects like palatal 
movements of the molars and tipping of the dental crowns which were seen with traditional Pendulum aplliance.

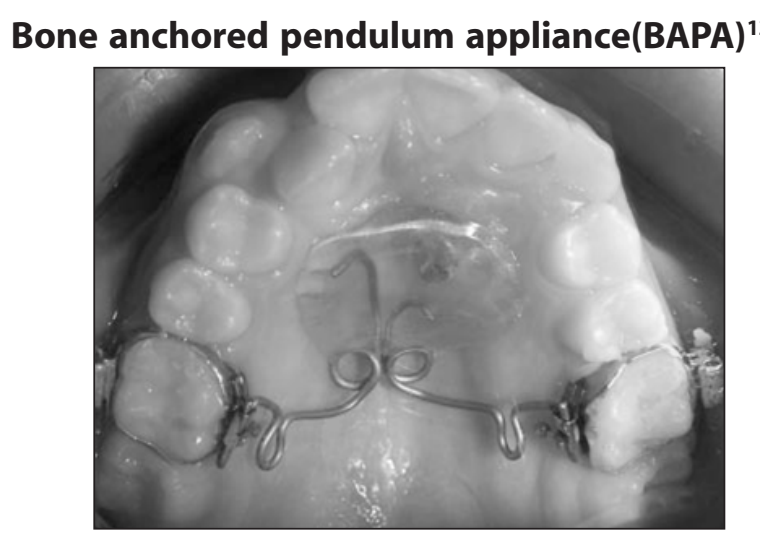

Intraoral distalization appliances have been designed to deliver a continuous reciprocal force on the maxillary first molars. Any action to move molars distally produces a mesial reaction force on the anchoring teeth. As a consequence, if the premolars or incisors (or both) are the anchoring teeth, they move mesially, the incisors protrude, and overjet increases. However, this effect is in contradiction with the main objective of Class II treatment. Furthermore, distalized molars are questionable anchors for the retraction of premolars and incisors.

Recently, researchers have tried to overcome this major problem by designing new intraoral systems involving rigid skeletal anchorage. Byloff et al designed the Graz implant-supported pendulum appliance in year 2000.

Beyza et al ${ }^{12}$ has designed BAPA which uses a titanium intraosseous screw $(2.0 \mathrm{~mm}$ diameter 38 $\mathrm{mm}$ length) (IMF intermaxillary fixation screw, Stryker, Leibinger, Germany) as a rigid bone anchor. The screw was inserted in the anterior paramedian region of the median palatal suture, $7-8 \mathrm{~mm}$ posterior to the incisive foramen and 3-4 mm lateral to the median line. It was found that molar distalization, as well as premolar distalization, was achieved with BAPA without any anchorage loss.

The BAPA presented an effective and minimally invasive, compliance-free alternative for intraoral molar distalization in nonextraction Class II treatment.

\section{Conclusion:}

Among the methods described in the recent literature for nonextraction treatment of a Class II malocclusion by molar distalization, the Hilgers Pendulum Appliance seems to best satisfy the most important requirements:

- Avoidance of undesirable biomechanical side effects.

- Minimization of the need for patient cooperation.

- Acceptability in terms of esthetics and function.

- Economy of chairtime for placement and reactivations.

With so many modifications available it can be best used to advantage for a particular clinical situation to achieve desirable results.

\section{References:}

1. Ucem T.T.Effects of a three dimensional biometric maxillary distalizing arch. Eur J Orthod 2000;22:293-298

2. Abu A.Joseph and Chris J. Butchart .An Evaluation of the Pendulum distalizing appliance Semin orthod 2000;6:129-135.

3. Scuzzo G, Kyoto Takemoto, Flavio Pisani Maxillary molar distalization with a modified pendulum appliance. J Clin Orthod $1999 ; 33:$ 645-650

4. Hilgers J.J. The Pendulum appliance for class II Noncompliance therapy. J Clin Orthod 1992;26: 706-714.

5. Ghosh J, Nanda RS. Evaluation of an intraoral maxillary molar distalization technique. Am J Orthod Dentofac Orthop 1996;110:639-646.

6. BussickTJ and McNamara JA ,Jr. Dentoalveolar and skeletal changes associated with the pendulum appliance, Am J Orthod Dentofac Orthop 2000;117:333-343.

7. Friedrich K. Byloff, Ali Darendeliler \&Ernst clar. Distal molar movement using the Pendulum appliance Part II; The effects of maxillary molar root uprighting bends. Angle Orthod 1997;67:261-270

8. Scuzzo G, Kyoto Takemoto, Flavio Pisani . The Modified Pendulum appliance with Removable arms J Clin Orthod 2000; 34:244-246

9. Byloff F. Mandibular molar distalization with the Franzulum appliance. J Clin Orthod 2000;34:518-523

10. James J.Hilgers \& Stephen G.Tracey. The Mini-Distalizing Appliance: The Third Dimension in Maxillary Expansion. J Clin Orthod 2003;37:467-475.

11. Gero S. M. Kinzinger, Dr med dent, Ulrike B. Fritz. Efficiency of a pendulum appliance for molar distalization related to second and third molar eruption stage. Am J Orthod Dentofacial Orthop 2004;125:8-23

12. Beyza Hancolu Kircelli, Zafer Özgür Pekta, and Cem Kircelli. Maxillary molar distalization with a Bone Anchored Pendulum Appliance. Angle Orthod 2006;76:4,650-659. 